\title{
Ligaçôes perigosas: indústria farmacêutica, associaçôes de pacientes e as batalhas judiciais por acesso a medicamentos
}

\section{| 1 Jussara Calmon Reis de Souza Soares, ${ }^{2}$ Aline Scaramussa Deprá I}

Resumo: O presente trabalho busca trazer novos elementos para o debate sobre a judicialização da saúde e sobre o acesso a medicamentos. Parte-se de um breve panorama do setor farmacêutico, seguido de um levantamento bibliográfico sobre demandas judiciais por medicamentos no Brasil, para fundamentar uma reflexão sobre as ligações entre a indústria farmacêutica e as associaçôes de pacientes, que podem estar interferindo no processo. Tais ligações resultam das novas estratégias de expansão do mercado pelo setor farmacêutico, e podem agravar o panorama da saúde brasileira em relação ao uso crítico e responsável dos medicamentos. A necessidade de desmercantilização da saúde é incluída nesse debate.

> Palavras-chave: indústria farmacêutica, ONGs, pacientes, uso de medicamentos, decisões judiciais.

\author{
1 Professora Associada do \\ Departamento de Saúde \\ e Sociedade do Instituto \\ de Saúde da Comunidade, \\ Universidade Federal \\ Fluminense; pesquisadora do \\ Grupo de Estudos de Gerência \\ e Ensino em Saúde - GEGES/ \\ UFF. Endereço eletrônico: \\ jucalmon@vm.uff.br \\ ${ }^{2}$ Farmacêutica do Corpo de \\ Bombeiros Militar do Estado do \\ Rio de Janeiro; mestranda em \\ Saúde Coletiva (PPGSC/UFF). \\ Endereço eletrônico: aline_. \\ uffarma@yahoo.com.br
}


Medicamentos estão entre as tecnologias mais utilizadas pelo setor saúde, e são considerados essenciais para a prática da medicina ocidental contemporânea (CAMARGO JR., 2005). São também importantes indicadores sanitários e culturais, e trazem contribuições valiosas à compreensão das relaçôes de consumo no campo da saúde. Com o avanço do processo de mercantilização no setor, as tecnologias utilizadas tornaram-se fins, e passaram a atender mais a interesses econômicos do que às necessidades de saúde das populaçôes. No Brasil - $9^{\circ}$ maior consumidor mundial de medicamentos per capita - entre $15 \%$ e $20 \%$ da população não têm acesso a qualquer tipo de medicamento, e $50 \%$ dos pacientes que dele precisam, não dispõem de meios para comprá-lo. Nas farmácias privadas, $15 \%$ da população mais rica consomem $48 \%$ dos medicamentos e $51 \%$ da população, os mais pobres, consomem apenas 16\% (CREMESP; CRFSP; IDEC, 2006).

Em nível mundial, o padrão de consumo é ainda mais perverso: segundo dados da OMS, em 1999, os países de maior renda - apenas 15\% da população mundial - consumiam cerca de $90 \%$ dos medicamentos produzidos no planeta. Só os EUA passaram de 18,4\% do consumo total mundial, em 1976, para mais de 52\%, em 2000 (WHO, 2004). Ou seja, a grande maioria da população não tem acesso a medicamentos, enquanto uma pequena parcela $-15 \%$, vale repetir - consome $90 \%$ da produção global. Observam-se, no cenário internacional, iniciativas diversas em prol da garantia de acesso, principalmente para usuários de países periféricos, em sua maioria, excluídos desse direito (WHO, 2007).

No caso brasileiro, o acesso é colocado como pedra angular da Política Nacional de Medicamentos, na qual se afirma que os gestores do Sistema Único de Saúde, nas três esferas de Governo, devem assegurar à população medicamentos seguros, eficazes e de qualidade, ao menor custo possível (BRASIL, 1998). Na prática, essa meta está muito longe de ser atingida. Embora o governo tenha aumentado consideravelmente seus gastos totais com saúde em 9,6\% - e em 123,9\% com medicamentos - entre 2002 e 2006 (BRASIL, 2007), a situação ainda é muito diferente da encontrada nos países centrais com sistemas universais de saúde, nos quais os governos arcam com no mínimo $70 \%$ desses gastos. No Brasil, eles pesam mais no bolso das famílias brasileiras do que no orçamento do governo: em 2007, os brasileiros gastaram $\mathrm{R} \$ 45$ bilhôes com a compra de medicamentos, cerca de dez vezes mais do que o governo, no mesmo ano (IBGE, 2009). 
Os gastos com medicamentos sobem em todos os países. Números divulgados pelo Department of Health \& Human Services mostram que, nos EUA, esses gastos passaram de 96 dólares por pessoa, em 1980, para 709 dólares em 2003. Na França, esse valor ficou em 572 dólares por pessoa em 2003 (COGHLAN, 2007). Ainda em relação aos norte-americanos, segundo Angell (2008), eles gastam 200 bilhões de dólares por ano com medicamentos vendidos sob prescrição médica, e esse valor cresce a uma taxa de $12 \%$ ao ano.

No Brasil, o direito à saúde é garantido constitucionalmente por meio do acesso universal e igualitário às ações e serviços para a sua promoção, proteção e recuperação (BRASIL, 2009a). Porém, como descrito acima, a assistência farmacêutica ainda é limitada. Há atualmente um aumento exponencial das ações judiciais por acesso a medicamentos. Até junho de 2009 foram impetradas no Supremo Tribunal Federal (STF) 2.762 ações referentes ao tema, segundo informe da Secretaria de Ciência, Tecnologia e Insumos Estratégicos do Ministério da Saúde (BRASIL, 2009b). Esse processo, chamado de judicialização da saúde, vem causando enormes prejuízos financeiros, além de potenciais danos à saúde dos pacientes, pela utilização inadequada de medicamentos (MARQUES; DALLARI, 2007; CHIEFFI; BARATA, 2009). O Ministério da Saúde destinou aproximadamente $\mathrm{R}$ \$ 2 milhões de seu orçamento para atender às sentenças judiciais em 2005, valor que saltou para R \$ 68 milhões em apenas três anos (BRASIL, 2009b).

As lacunas deixadas pelas políticas públicas muitas vezes inexistentes ou insuficientes fazem com que a população, de forma justificada e legitima, busque os meios jurídicos para conseguir seu tratamento. É fundamental que o Estado cumpra seu papel em relação às reais necessidades de assistência à saúde no país. Porém, é igualmente importante avaliar até que ponto outros interesses podem estar também interferindo no processo de judicialização, causando mais danos do que benefícios à saúde da população brasileira. Como afirmou o ministro da saúde José Gomes Temporão, o ministério se posicionou contrário à adoção, por medida judicial, de novas tecnologias, insumos ou medicamentos sem evidência científica e sem registro na Agência Nacional de Vigilância Sanitária (BRASIL, 2009).

Faz-se urgente aprofundar a análise desse processo. $\mathrm{O}$ presente trabalho busca compartilhar reflexóes e trazer novos elementos para o debate sobre o tema do acesso a medicamentos, a inovações tecnológicas, a partir de levantamentos bibliográficos realizados para fundamentar e contextualizar uma pesquisa em 
andamento, que discute as estratégias utilizadas pelas indústrias farmacêuticas junto a associaçōes de pacientes no Brasil (DEPRÁ, 2010). Apresentamos um breve panorama do setor farmacêutico, seguido de um levantamento bibliográfico exploratório sobre demandas judiciais por medicamentos no Brasil. Refletimos, então, sobre as ligações entre a farmaindústria e a população leiga, com foco nas associações de pacientes. Esse tema, que já vem sendo bastante pesquisado em diversos países, é ainda incipiente entre nós. Por meio deste trabalho, buscamos contribuir com novos elementos para o debate sobre a judicialização da saúde e, em um nível mais geral, sobre o acesso a medicamentos.

\section{A farmaindústria}

O setor farmacêutico está entre os mais globalizados e lucrativos do mundo. Há cerca de 20 anos, o mercado mundial de medicamentos movimentava aproximadamente 12 bilhões de dólares. Em 2001, atingiu US $\$ 393$ bilhões (IMS HEALTH, 2008). Desde então houve um crescimento de quase $200 \%$, alcançando, em 2008 - quando o mercado farmacêutico global cresceu 4,8\% ao ano - um montante de 773 bilhōes de dólares em vendas. De acordo com as estimativas, essa tendência será mantida e, até 2013, devem-se alcançar 975 bilhões (IMS HEALTH, 2010). A farmaindústria segue em crescimento contínuo - em todos os países europeus, cresceu mais que o PIB nos últimos 20 anos (ESS; SCHNEEWEISS; SZUCS, 2003) -, e tem entre as principais chaves para o sucesso de vendas, a promessa da novidade, da inovação tecnológica.

Embora o setor argumente que os preços elevados dos medicamentos devem-se aos investimentos em Pesquisa \& Desenvolvimento (P\&D) - cerca de US\$800 milhões por medicamento -, levantamento realizado pelo Health Research Group estimou que o valor real estivesse entre 71 e 150 milhões de dólares (ANGELL, 2008). Soares (2005) apresentou estudos que mostram que a farmaindústria gasta mais com marketing e administração. Public Citizen (2003) trouxe dados sobre esses gastos. Gadelha (1990) afirmou que empresas líderes do setor destinam entre $10 \%$ e $20 \%$ de seu faturamento às atividades de P\&D e 40\% com marketing (apud GADELHA; QUENTAL; FIALHO; 2003). Como a inovação é cada vez mais difícil, e com a forte concorrência no setor, a "promoção farmacêutica" cresce em importância, atingindo um gasto médio de $35 \%$ do valor das vendas (SOARES, 2008). 
Também são já bastante conhecidas as inúmeras estratégias de propaganda e marketing utilizadas pelo setor, nos mais diversos meios e veículos de comunicação (BARROS, 1995; LEXCHIN, 1997; SOARES, 2008). Além dos propagandistas, outras vias utilizadas incluem a promoção de palestras por especialistas considerados opinion makers, a inserção de matérias sobre novos medicamentos, novos procedimentos e novas doenças em jornais e revistas (MINTZES, 2006). Como afirmou Coons (2003), basta olhar as páginas dos jornais norte-americanos para constatar a presença marcante de manchetes relativas a medicamentos, as chamadas para ensaios clínicos, até mesmo tiras de humor, sem contar as propagandas. $\mathrm{O}$ mesmo pode ser observado no Brasil e em vários outros países, e não apenas nos jornais, mas também, em revistas, programas de televisão e de rádio, etc.

$\mathrm{Na}$ produção acadêmica sobre propaganda de medicamentos nos EUA e Europa, destaca-se, hoje, a propaganda direta ao consumidor (DTCA), como mostram, por exemplo, as revisões da literatura por Auton (2004; 2006). Em 2008, Sillup e Porth (2008) analisaram a cobertura jornalística norte-americana sobre questões éticas na indústria farmacêutica, e realizaram uma extensa revisão bibliográfica com um panorama dos problemas praticados pelo setor. Entre os pontos levantados, há o chamado processo de disease mongering, a "redefiniçãa" de doenças, assim como a elaboração de novas diretrizes terapêuticas alterando os limites de normalidade para ampliar o número de pacientes a tratar; foram citados, ainda, os usos de organizaçooes de pesquisa clínica, de ghostwriters para publicação mais rápida de resultados favoráveis às indústrias, além de recompensas financeiras a centros clínicos por parte do setor farmacêutico.

Há, também, exemplos de decisões de agências reguladoras que atendem aos interesses econômicos do lobby farmacêutico. Um inquérito realizado, em 2002, pelo $D H H S$, no qual foram entrevistados quase 400 pesquisadores do FDA, mostrou que $18 \%$ deles se sentiram pressionados a aprovarem novos medicamentos ou novos usos para produtos. 36\% afirmaram ter pouca ou nenhuma confiança nas decisões do FDA sobre segurança dos medicamentos. Em relação à eficácia, o índice caiu para 22\%. Apenas 6\% afirmaram ter total confiança na capacidade de vigilância pós-comercialização da agência americana acerca da segurança dos medicamentos de venda sob prescrição. Por fim, mais da metade dos profissionais entrevistados afirmou não ter tido tempo adequado 
para a condução das revisões necessárias, no caso de produtos considerados prioritários (KAISER DAILY HEALTH POLICY REPORT, 2004).

Com a crescente utilização da internet, esse meio de comunicação tornou-se importante ferramenta de marketing farmacêutico, inclusive por meio de sites de relacionamento tais como Myspace e Facebook (WEBB, 2010). Sweet (2009) apresentou vários exemplos de estratégias de promoção farmacêutica pela internet para influenciar as expectativas de consumidores em relação à atenção em saúde e aos medicamentos; empresas farmacêuticas vêm criando e utilizando blogues e websites para promover seus produtos, assim como para fortalecer as relaçôes com os consumidores. Ou seja, embora os médicos continuem sendo o alvo principal das propagandas e do marketing da indústria farmacêutica, cresce a busca de influência direta junto à população leiga. Percebem-se, por exemplo, as tentativas de ampliação da aprovação da DTCA, propaganda de medicamentos de venda sob prescrição, diretamente ao consumidor. Atualmente, apenas os EUA e a Nova Zelândia a permitem. Em 2007, cinco associaçôes internacionais - Health Action International (HAI), International Society of Independent Drug Bulletins, European Consumers' Organization, Medicines in Europe Forum e Association Internationale de La Mutualité - precisaram manifestar-se junto ao European Commission Pharmaceutical Forum, a fim de impedir ações que previam o afrouxamento das regras para propaganda ao público (EUROPE..., 2007).

Nesse processo de estreitamento das relaçôes da farmaindústria com a população incluem-se, obviamente, as associações representativas de pacientes, de consumidores de medicamentos, na busca pela intervenção nas políticas de medicamentos e de saúde, no sentido de atender aos interesses comerciais do setor farmacêutico, como bem demonstraram, por exemplo, Ball, Tisocki e Herxkeimer (2006). Sweet (2009) citou uma campanha através da internet, dirigida pelo setor farmacêutico, em que foram obtidas mais de 80 mil assinaturas em apenas três semanas, em uma petição para influenciar as decisões sobre financiamento de vacinas contra papilomavirus. Tais ações da farmaindústria trazem novos e importantes desafios para gestores e agências reguladoras, uma vez que seu controle e regulamentação são muito difíceis, dadas as características desse meio de comunicação. 


\section{da saúde: o caso dos medicamentos}

O termo refere-se ao crescente fenômeno da busca, por meio da Justiça, de medicamentos ou demais tecnologias que os usuários não conseguem obter diretamente no Sistema Único de Saúde. Esse processo iniciou-se com a demanda por medicamentos antirretrovirais para a Aids. Por um lado, impetrar ações judiciais pode ser um indicador do aumento da consciência de que a saúde é um direito do cidadão e um dever do Estado. Por outro, porém, constata-se atualmente um crescimento contínuo das solicitações de medicamentos.

$\mathrm{Na}$ Bahia, o primeiro caso de judicialização ocorreu em 2002. Em 2006, foram 34 e, em 2007, 112 açóes. Muitas demandas eram para o atendimento oncológico de pacientes que haviam iniciado seu tratamento em clínicas privadas. Só em 2008, foram gastos mais de 5 milhōes de reais para atender a esses casos. Em Santa Catarina, o assessor jurídico da Secretaria Estadual de Saúde relatou que, enquanto em 2002 registraram-se apenas 24 ações, cinco anos depois esse número subiu para 2.511. Segundo Batalha, mais de $50 \%$ dos valores despendidos com ações foram para atendimentos a pacientes oncológicos.

Os medicamentos importados são outra fonte importante de demandas judiciais naquele estado do sul brasileiro. No Rio Grande do Sul, um dos primeiros estados brasileiros a apresentar casos de judicialização, havia, naquele ano, mais de 18 mil pacientes ativos demandando tratamentos especiais (ENSP, 2008).

Borges e Ugá (2010) analisaram as ações judiciais individuais para fornecimento de medicamentos, propostas por usuários do SUS contra o Estado do Rio de Janeiro, no ano de 2005. Entre 2001 e 2005, o número de ações havia aumentado aproximadamente $350 \%$ no estado. Os dados obtidos indicaram, segundo as autoras, tanto a falta de adoção de critérios pelo poder judiciário na questão do fornecimento de medicamentos, quanto falhas na prestação da assistência farmacêutica pelo poder executivo.

A correta utilização dos medicamentos deve atender a critérios que incluem a comprovação de sua eficácia e segurança, relação positiva benefício/risco e relação benefício/custo favorável. É fundamental o seu registro na ANVISA, agência 
regulatória nacional, para que eles possam ser comercializados no país. Também devem ser estabelecidas normas para a inclusão e exclusão de medicamentos no rol de produtos ofertados à população pelo SUS. Além da Relação Nacional de Medicamentos Essenciais (RENAME), o Ministério da Saúde vem aprovando protocolos clínicos e diretrizes terapêuticas para fundamentar as padronizações de medicamentos nos diferentes níveis de atenção. Entretanto, como constataram Chieffi e Barata (2009), a interferência do poder judiciário em questões que são, primariamente, da competência dos poderes executivos ou legislativos torna-se cada vez mais frequente. As ações judiciais passaram a ocupar lugar de destaque na assistência farmacêutica, e desrespeitam, muitas vezes, os princípios que deveriam reger a utilização de medicamentos.

A transferência de recursos previamente designados ao provimento das políticas públicas para o cumprimento de mandados judiciais pode agravar ainda mais a escassez de medicamentos na rede própria do SUS, haja vista a limitação dos recursos e a necessidade de planejamento estratégico. Como destacaram Marques e Dallari (2007, p. 105), o judiciário não deve ficar à mercê de açōes que podem "[...] acobertar os interesses de determinados laboratórios farmacêuticos, responsáveis pela comercialização de inovações terapêuticas inacessíveis financeiramente aos autores."

A condução das ações judiciais contra a SES-RJ em 2005 foi feita, em 20,3\% dos casos, por escritórios particulares de advocacia e, destes, grande parte é integrante de associações de portadores de condições patológicas específicas ou de organizações não governamentais, (MESSEDER; OSÓRIO-DE-CASTRO; LUIZA, 2005). Tal fato veio reforçar a hipótese de que, também no Brasil, o setor farmacêutico venha estreitando as relações com as associações de pacientes.

Conforme levantamento feito recentemente pelo próprio Ministério da Saúde, em $60 \%$ dos processos judiciais a demanda é por produtos não disponíveis no SUS, embora os tratamentos em questão pudessem ser realizados com medicamentos oferecidos pelo Sistema. As demais ações referem-se a drogas de última geração tecnológica - muitas ainda sem registro na ANVISA - ou seja, que não tiveram sua segurança e eficácia comprovadas no país (BRASIL, 2009c).

Paradoxalmente, o que poderia ser sinal de conscientização e de ampliação de cidadania - a busca da Justiça para o acesso a medicamentos, como parte da garantia do direito à saúde -, tornou-se um problema de saúde pública, e está em 
foco no cenário político brasileiro. Tendo em vista as sérias consequências desse aumento, vem ocorrendo no Brasil uma intensa mobilização no sentido de um aprofundamento do debate e de um maior entendimento entre os diversos poderes e sujeitos envolvidos. Além das pesquisas realizadas, diversos eventos e fóruns de discussão ocorreram recentemente. Em maio de 2009, por exemplo, houve, no Senado Federal, o Fórum Medicamentos, Políticas Públicas e Judicialização da Saúde. Discutiu-se, entre outros assuntos, o projeto de lei no 219 de 2007 - do Senado Federal - que estabelece a oferta de medicamentos pelo SUS somente com base em prescrições amparadas em protocolos clínicos aprovados pelo Ministério da Saúde. Em abril de 2010, o Conselho Nacional de Justiça decidiu instituir o Fórum Nacional do Judiciário, para monitoramento e resolução das demandas de assistência à saúde, por meio da Resolução no 107/ 2010 (BRASIL, 2010).

Mesmo em se tratando de demandas legítimas de determinados grupos, há que se avaliar até que ponto a luta por interesses particulares de grupos específicos pode ou deve se sobrepor aos interesses coletivos da grande maioria da população. Como bem enfatizou Boaventura de Souza Santos (2010), o ativismo judicial pode trazer avanços ou retrocessos. Outro ponto de grande preocupação é o deslocamento da legitimidade do poder executivo para o judiciário. É fundamental, portanto, enfrentar o desafio de saber e poder diferenciar, no processo, ações que possam de fato trazer maior garantia aos direitos de cidadania no Brasil, daquelas que apenas visam à expansão de interesses de mercado. Trata-se de garantir o direito legítimo e fundamental de acesso aos medicamentos e demais ações e serviços no sentido da promoção da saúde, e não de lutar pelo direito ao consumo de mercadorias em nome da saúde.

No presente trabalho, importa ressaltar as indicações de que o setor farmacêutico possa estar se valendo de novos caminhos para influenciar a utilização de medicamentos inovadores - incluindo experimentais - no tratamento de diversas doenças, por meio do incentivo à impetração de ações judiciais como forma de acesso aos novos fármacos ou da pressão por sua entrada nos protocolos clínicos aprovados para inserção no SUS. Os produtos são apresentados aos doentes e seus familiares como a melhor tecnologia e, em alguns casos, como a única forma possível de tratamento, muitas vezes estimulado pelos próprios médicos. Os indícios apontam para uma possível atuação dos fabricantes de medicamentos por meio da influência que exercem junto a associações de pacientes, o que 
evidencia a necessidade urgente de se aprofundar esse tema e de se refletir sobre seu impacto para a saúde e para o SUS.

\section{Algumas evidências das ligações entre ONGs e a farmaindústria}

Mosconi (2003) constatou a falta de transparência em relação ao patrocínio financeiro por indústrias farmacêuticas em cerca de cem das associações de câncer de mama pertencentes ao fórum italiano Europa Donna. 1/3 das 67 associações que responderam ao questionário sobre financiamento havia recebido recursos de laboratórios, e, entre elas, apenas duas haviam declarado conflito de interesses. Para a pesquisadora, esses dados podem comprometer a independência das associaçóes de pacientes e de consumidores. Para a autora, existe a necessidade de mais financiamento público para as associações que participam do debate em saúde pública.

Outros pesquisadores confirmam a extensão dessa dependência entre ONGs e indústrias. Lenzer (2003) afirmou que The National Prostate Cancer Coalition havia admitido que $95 \%$ dos seus recursos financeiros vêm de diversos laboratórios farmacêuticos. Herxheimer (2003) apresentou um panorama das associaçôes de pacientes no Reino Unido, confirmando a frequente parceria com as indústrias farmacêuticas. Mostrou como várias associações são usadas como parte da estratégia dos laboratórios para influenciar autoridades nos EUA e Reino Unido. Observou, ainda, que diversas foram criadas com a participação do próprio setor farmacêutico, tais como a International Association of Patient Organizations (IAPO), a Global Alliance of Mental Illness Advocacy (GAMIAN) e a World Parkinson's Disease Association.

Em 2006, Ball, Tisocki e Herxheimer (2006) avaliaram os conflitos de interesse entre associações de pacientes e a farmaindústria. Os autores pesquisaram 69 homepages de organizações de pacientes - nacionais e internacionais - a fim de constatar a declaração, ou não, de suporte financeiro das mesmas por laboratórios farmacêuticos. Apenas quatro dos 69 sites afirmaram a existência de conflitos de interesse. $45 \%$ das organizações declararam financiamento pela farmaindústria, mas não foi possível avaliar sua extensão em relação aos recursos totais das associações. Embora não houvesse propaganda na maioria dos sites (até porque a DTCA é ilegal na grande maioria dos países), em 1/3 deles havia logomarcas e/ou links para as páginas dos laboratórios patrocinadores. 
No mesmo ano, Marshall e Aldhous (2006) apresentaram os resultados do maior inquérito realizado até aquela data, pela New Scientist, sobre as doações da farmaindústria a associações de pacientes americanos. A extensão do patrocínio variou bastante, com casos de mais de 23 milhões de dólares para a American Heart Association - que foi a maior organização estudada -, embora essa quantia representasse apenas $4 \%$ do seu financiamento total. Sete grupos receberam $20 \%$ ou mais de seus patrocínios de empresas farmacêuticas ou de equipamentos médicos. De todos os grupos identificados no inquérito, apenas dois - The National Women's Health Network (NWHN) e a Breast Cancer Action - se recusavam a aceitar tais doações.

Além de denunciar o poderoso lobby da farmaindústria nos EUA e os financiamentos de campanhas políticas, Angell também faz referência ao patrocínio de "[...] uma série de grupos de fachada que se passam por organizaçôes autênticas. Um deles se chama Citizens for Better Medicare [...]” (ANGELL, 2008, p. 215).

Observa-se, de um lado, a farmaindústria, que, como qualquer outro ramo comercial, visa à expansão de seu mercado. Para isso, as empresas buscam apoio e fazem lobby contra restrições impostas por políticas governamentais e institucionais. De outro, as associações de pacientes, que necessitam de apoio para os projetos desenvolvidos, produção de materiais informativos e, principalmente, financiamento para suas ações. Elas afirmam a importância em manter relações de cooperação com empresas que desenvolvem novos medicamentos, de modo a fomentar a comunicação entre seus pacientes e os fabricantes, uma vez que as decisões afetam diretamente o tratamento de seus associados.

Para alguns pesquisadores, é aceitável que as organizações recebam doações financeiras das companhias, contanto que essa relação seja transparente, e que não possa afetar o bem-estar dos pacientes. Entretanto, este parece ser um ponto importante de debate: é possível conciliar o recebimento de patrocínio financeiro e a independência nas decisões e propostas de ação? Embora o patrocínio financeiro de associações de pacientes por indústrias farmacêuticas seja bastante frequente em muitos países, em função do claro conflito de interesses aí presente, a dependência financeira mina a imparcialidade das decisões e ações dos grupos de apoio a pacientes (MINTZES, 2007).

Embora não haja pesquisas publicadas no Brasil comprovando esse processo, encontramos indícios de sua ocorrência em nosso país. Em maio de 2008, a 
61 Assembléia Mundial da Saúde, que aprovou a Resolução WHA 61.21, foi resultado de reuniōes do Intergovernmental Working Group on Public Health, Innovation and Intellectual Property (IGWG), para discutir formas de promover a inovação e a capacidade de maior acesso aos produtos de saúde nos países em desenvolvimento. $\mathrm{O}$ trabalho foi complementado por diversas contribuições feitas a partir de duas audiências públicas, via web. Entre as propostas encaminhadas, estava um manifesto internacional organizado pela ONG Patients and Patents, denominado Patient Declaration on Medical Innovation and Access, absolutamente favorável à manutenção do sistema de patentes de medicamentos na forma então vigente, seguindo a posição defendida pela indústria farmacêutica.

Por sua vez, o estudo realizado pela ONG Essential Action, em 2008, sobre o referido documento, mostra que 61 das mais de 110 entidades signatárias apresentavam ligações com indústrias farmacêuticas ou com fabricantes de equipamentos médicos. Delas, nove são brasileiras, e três destas afirmam dedicar-se ao controle do câncer. Além disso, Chieffi e Barata (2010) em sua análise de ações judiciais em São Paulo e com base no trabalho de Angell (2008), mencionam a existência de lobby da indústria com associações de portadores de doenças crônicas.

Assim como em todas as situações já bastante conhecidas de envolvimento entre laboratórios farmacêuticos e pesquisadores, acadêmicos e médicos, as organizaçoos representativas de pacientes também afirmam que suas atividades não são subordinadas aos interesses dos laboratórios patrocinadores. Em matéria sobre as relações financeiras entre ONGs ligadas ao tratamento do câncer e indústrias farmacêuticas no Brasil, os repórteres Cláudia Collucci e Ricardo Westin, do jornal Folha de São Paulo, colheram depoimentos que confirmam tal atitude: "Só dão o dinheiro. Não somos agentes da indústria para vender medicamentos" - Associação Brasileira do Câncer -; "Não há nenhuma contrapartida" - Associação Brasileira de Linfoma e Leucemia. Porém, como constataram os jornalistas, ao menos $70 \%$ do orçamento anual da ABCâncer, cerca de R \$ 936 mil, vêm de cinco laboratórios. Já a Abrale recebeu, em 2007, R\$1,5 milhão de oito laboratórios (COLLUCCI; WESTIN, 2008).

A indústria farmacêutica, evidentemente, não reconhece conflito de interesses entre o fato de associações de pacientes serem financiadas por laboratórios e, ao mesmo tempo, apoiarem causas defendidas por essas instituições, tais 
como a questão das patentes e muitas outras. $\mathrm{O}$ que parece mais grave é que pacientes tampouco questionam tais relações. Como constataram Hampson e colaboradores (2006), participantes de ensaios clínicos em câncer não veem anormalidade nas ligações financeiras entre pesquisadores, centros médicos e indústrias farmacêuticas. Os autores entrevistaram 253 pacientes inscritos em pesquisas clínicas sobre câncer de cinco institutos nos EUA, entre novembro de 2004 e novembro de 2005: mais de 90\% não consideraram esse fato um problema, e a grande maioria afirmou ser ético o recebimento de pagamento feito pelos laboratórios aos pesquisadores que dessem consultoria ou proferissem palestras sobre seus produtos. Ainda segundo os pesquisadores, a saúde e a obtenção do melhor cuidado eram fatores mais importantes, para os pacientes, do que as ligações financeiras entre indústria e pesquisa. Aliás, no Brasil, também cresce a cada dia a venda de medicamentos à população, através dos sites dos fabricantes, com ofertas, descontos, programas de fidelização de clientes, etc.

\section{À guisa de conclusão}

Como dito no início deste artigo, o processo de judicialização da saúde no Brasil vem causando enormes prejuízos financeiros, além de potenciais danos à saúde dos pacientes, pela utilização inadequada de medicamentos. Apenas em 2007, São Paulo gastou 400 milhões de reais em medicamentos, para 25 mil pessoas. Segundo matéria publicada no jornal Folha de São Paulo, em 2009, as ações judiciais para a aquisição de medicamentos consumiram $\mathrm{R} \$ 52$ milhões do Ministério da Saúde em 2008, o triplo do valor gasto em 2007. Em três anos, o aumento dos custos com a judicialização foi de quase $2.000 \%$ (COLLUCCI, 2009).

Chieffi e Barata (2009) avaliaram os processos judiciais referentes a medicamentos, movidos por moradores da cidade de São Paulo, no ano de 2006. Dos 954 medicamentos diferentes solicitados, 3\% não estavam disponíveis para venda no mercado nacional e $77 \%$ não pertenciam aos programas de assistência farmacêutica do SUS. Entre os itens fornecidos pelo SUS (23\% do total), 13\% apenas pertenciam ao Programa de Medicamentos de Dispensação Excepcional. A transferência de recursos previamente designados ao provimento das políticas públicas, para o cumprimento de mandados judiciais, agrava a escassez de medicamentos na rede própria do SUS, haja vista a limitação dos recursos e a necessidade de planejamento baseado nas políticas de saúde, para sua utilização. 
Está nas mãos do Judiciário brasileiro a responsabilidade de julgar casos em que, em muitas vezes, prescriçôes médicas privilegiam medicamentos extremamente caros em situações em que o SUS oferece remédios eficazes para o mesmo tipo de tratamento a custo muito mais compatível. Impressiona e preocupa como a pressão pela incorporação de procedimentos experimentais, produtos não registrados no país, tecnologias sem forte consenso entre especialistas, que envolvem milhares de desdobramentos judiciais, têm distorcido a imagem da gestão do SUS, incorretamente tratada como dificultadora do acesso a procedimentos e medicamentos. Transferir para o SUS a responsabilidade por atendimento realizado fora de suas normas operacionais pode gerar conseqüências como a desregulação do acesso assistencial, perda da integralidade e redução de controle e avaliação da atenção prestada. (TEMPORÃO, 2009, p.2)

É fundamental aprofundar os conhecimentos sobre as relações existentes entre o setor farmacêutico e as associaçôes representativas de portadores de doenças e os advocacy groups, uma vez que podem agravar o panorama da saúde brasileira em relação ao uso crítico e responsável dos medicamentos. Em um levantamento preliminar foi possível listar 80 ONGs relacionadas a pacientes com câncer (DEPRÁ, 2010). Como vem se dando o acesso aos grupos no Brasil? Quais as principais estratégias utilizadas? Estão influenciando os processos de judicialização da saúde no Brasil? Qual o impacto, para a saúde e para o SUS, de tais parcerias? Essas são algumas questões importantes e urgentes para o debate político sobre o acesso aos medicamentos, que não pode ignorar ou minimizar o peso das interferências causadas pela farmaindústria. E, evidentemente, a reflexão sobre a necessidade de desmercantilização da saúde deve estar no âmago desse debate. ${ }^{1}$

\section{Referências}

ANGELL, M. A verdade sobre os laboratórios farmacêuticos. 3 ed. Rio de Janeiro: Record, 2008.

AUTON, F. Direct-to-consumer advertising of pharmaceuticals: an updated review of the literature and debate since 2003. Institute of Economic Affairs, v.26, n.3, p.24-32, 2006.

. The advertising of pharmaceuticals direct to consumers: a critical review of the literature and debate. International Journal of Advertising, v. 23, n.1, p. 23-52, 2004.

BALL, D.E.; TISOCKI, K.; HERXHEIMER, A. Advertising and disclosure of funding on patient organization websites: a cross-sectional survey. BioMed Central Public Health, v. 201, n. 6, 3 Aug. 2006. Disponível em: <http://www.biomedcentral.com/1471-2458/6/201>. Acesso em: 28 jul. 2009.

BARROS, J.A.C. Propaganda de medicamentos: atentado à saúde? São Paulo: Hucitec, 1995. 
BORGES, D.C.L.; UGÁ, M.L.D. Conflitos e impasses da judicialização na obtenção de medicamentos: as decisões de $1^{\mathrm{a}}$ instância nas ações individuais contra o Estado do Rio de Janeiro, Brasil, em 2005. Caderno Saúde Pública, v.26, n.1, p.59-69, 2010.

BRASIL. Conselho Nacional de Justiça. Resolução no 107 de 6/04/2010. Institui o Fórum Nacional do Judiciário para monitoramento e resolução das demandas de assistência à saúde. Diário da Justiça Eletrônico, Poder Legislativo, Brasília, DF, n. 61, 07 abr. 2010, p. 6-9. Disponível em: <http://www.cnj.jus.br/dje/djeletronico>. Acesso em: 16 set. 2010.

. Ministério da Saúde. Portaria no. 3.916/GM de 30 de novembro de 1998. Aprova a Política Nacional de Medicamentos. Diário Oficial [da] República Federativa do Brasil, Poder Executivo, Brasília, DF, 10 dez. 1998. Disponível em: <http://www.anvisa.gov.br/ legis/consolidada/portaria_3916_98.pdf>. Acesso em: 22 nov. 2009.

Ministério da Saúde. Secretaria Executiva. Área de Economia da Saúde e Desenvolvimento. Núcleo Nacional de Economia da Saúde. Evolução dos Gastos do Ministério da Saúde com Medicamentos. Brasília, DF, 2007.

- Ministério da Saúde. Informe Ciência, Tecnologia e Inovação em Saúde. Saúde é tema de audiência pública no Supremo Tribunal Federal. Edição Especial, v.6, n.1, jun.2009(b). Disponível em: <http://bvsms.saude.gov.br/bvs/periodicos/informe_CTI_ Judicializacao.pdf>. Acesso em: 16 mar. 2011.

. MinistériodaSaúde. PortalSaúde.MSapresentaaoSTFargumentoscontraajudicialização da oferta de medicamentos e insumos. Brasília, DF, 27 abr. 2009(c). Disponível em: <http:// portal.saude.gov.br/portal/aplicacoes/noticias/default.cfm?pg=dspDetalheNoticia\&id_ area $=124 \& C O \_N O T I C I A=10124>$. Acesso em: 05 nov. 2009.

BRASIL. Senado Federal. Secretaria Especial de Editoração e Publicações. Subsecretaria de Ediçôes Técnicas. Constituição da República Federativa do Brasil. Texto consolidado até a EC no 58 de 23/09/2009. Brasília, DF, 2009(a). Disponível em: http://www.senado.gov. br/legislacao/const/con1988/CON1988_23.09.2009/index.shtm. Acesso em: 16 set. 2010.

CAMARGO JR., K.R. A biomedicina. Physis: Revista de Saúde Coletiva, v.15(Sup), p.177201, 2005.

CHIEFFI, A.L.; BARATA, R.B. Judicialização da política pública de assistência farmacêutica e eqüidade. Caderno Saúde Pública, v.25, n.8, p.839-1849, 2009.

CHIEFFI, A.L.; BARATA, R.B. Ações judiciais: estratégia da indústria farmacêutica para introdução de novos medicamentos. Revista de Saúde Pública, v.44, n.3, p.421-9, 2010.

COGHLAN, A. US aims to break drug industry's hold on pricing. New Scientist Magazine, v. 2594, p. 8-9, mar. 2007. Disponível em: <http://www.newscientist.com/ article/mg19325943.800-us-aims-to-break-drug-industrys-old-on-pricing.html>. Acesso em: 10 dez. 2009. 
COLlUCCI, C. Triplicam as ações judiciais para obter medicamentos. Folha de São Paulo, São Paulo, 09 jan. 2009. Disponível em: <http://www.crfsp.org.br/joomla/index. php?option=com_content $\&$ view=article $\&$ id=1051: clipping-9012009\&catid $=42$ : clipping \&Itemid=60>. Acesso em: 10 jan. 2011.

COLLUCCI, C.; WESTIN, R. Indústria farmacêutica financia ONGs. Folha de São Paulo, 18 maio 2008. Disponível em: <http://wwwl.folha.uol.com.br/folha/cotidiano/ ult95u402991.shtml>. Acesso em: 25 jul. 2010.

CREMESP; CRFSP; IDEC. Medicamento: um direito essencial. São Paulo, 2006. Disponível em: <http://www.cremesp.org.br/library/modulos/publicacoes/pdf/cartilha_ medicamentos.pdf >. Acesso em: 08 abr. 2010.

COONS, S.J. Drugs in the headlines. Clinical Therapeutics, v.25, n.5, p.1500-2, 2003.

DEPRÁ, A.S. Conflitos de interesses nas demandas judiciais por medicamentos para tratamento de câncer no Brasil: o caso dos anticorpos monoclonais. 2010. Projeto de pesquisa (Mestrado em Saúde Coletiva) - Instituto de Saúde da Comunidade, Universidade Federal Fluminense, Niterói, 2010.

ESCOLA NACIONAL DE SAÚDE PÚBLICA. Projeto de Cooperação ENSP/SESDEC. Judicialização do acesso a medicamentos no Brasil em debate. Notícias ENSP, 24 jul. 2008. Disponível em: <http://www.ensp.fiocruz.br/portal-ensp/informe/materia/index. php? origem $=9 \&$ matid $=11953>$. Acesso em: 28 jul. 2010.

ESS, S.M.; SCHNEEWEISS, S.; SZUCS, T.D. European healthcare policies for controlling drug expenditure. PharmacoEconomics, v.21, n.2, p.89-103, 2003.

EUROPE reconsidering DTCA. Canadian Medical Association Journal, v.176, n.10, p.1405, 2007.

FOLHA DE SÃO PAULO. Triplicam as ações judiciais para obter medicamentos. Folha de São Paulo, 09/01/2009. Disponível em: < http://www.crfsp.org.br/joomla/index. php?option=com_content $\&$ view=article $\&$ id=1051: clipping-9012009\&catid $=42$ : clipping \&Itemid=60>. Acesso em: 10 jan. 2011.

GADELHA, C.A.G.; QUENTAL, C.; FIALHO, B.C. Saúde e inovação: uma abordagem sistêmica das indústrias da saúde. Cad. Saúde Pública, Rio de Janeiro, v.19, n.1, p.47-59, 2003. HAMPSON, L.A. et al. Patients' views on financial conflicts of interest in cancer research trials. The New England Journal of Medicine, v.355, n.22, p.2330-2337, 2006.

HERXHEIMER, A. Relationships between the pharmaceutical industry and patients' organisations. British Medical Journal, v.326, p.1208-1210, 2003.

INSTITUTO BRASILEIRO DE GEOGRAFIA E ESTATÍSTICA. Conta-Satélite de Saúde Brasil 2005 2007. Comunicação social, 09 dez. 2009. Disponível em: <http://www. ibge.gov.br/home/presidencia/noticias/noticia_impressao.php?id_noticia=1514>. Acesso em: 13 out. 2010 . 
IMS HEALTH . Global Pharmaceutical Sales, 2001-2008, 2008. Disponível em: <http:// www.imshealth.com/deployedfiles/imshealth/Global/Content/StaticFile/Top_Line_ Data/Global_Pharma_Sales_2001-2008_Version_2.pdf>. Acesso em: 20 out. 2009. . IMS Forecasts Global Pharmaceutical Market Growth of 4 - 6\% in 2010; Predicts 4 - 7\% Expansion Through 2013. Disponível em: <http://www.imshealth.com/portal/site/ imshealth/menuitem.a46c6d4df3db4b3d88f611019418c22a/?vgnextoid=500e8fabedf242 10VgnVCM100000ed152ca2RCRD\&cpsextcurrchannel=1>. Acesso em: 15 jan. 2010.

INSTITUTO NACIONAL DO CÂNCER. Patentes e indústria: a encruzilhada das ONGs. Revista Rede Câncer, v.6, p.34-35, 2008.

KAISER DAILY HEALTH POLICY REPORT. Dec. 16 2004. Disponível em: <http:// www.kaisernetwork.org>. Acesso em: 05 nov. 2009.

LENZER, J. Lay campaigners for prostate screening are funded by industry. British Medical Journal, v. 326, p. 680, 2003.

LEXCHIN, J. Uma fraude planejada: a publicidade farmacêutica no Terceiro Mundo. In: BONFIM, J.R.A.; MERCUCCI, V.L. (Org). A construção da política de medicamentos. São Paulo: Hucitec, 1997.

MARQUES, S.B.; DALLARI, S.G. Garantia do direito social à assistência farmacêutica no Estado de São Paulo. Revista Saúde Pública, v.41, p.101-7, 2007.

MARSHALL, J.; ALDHOUS, P. Patient groups special: Swallowing the best advice? New Scientist, v. 192, p.18-22, 2006.

MESSEDER, A.M.; OSORIO-DE-CASTRO, C.G.S.; LUIZA, V.L. Mandados judiciais como ferramentas para garantia do acesso a medicamentos no setor público: a experiência do Estado do Rio de Janeiro, Brasil. Cadernos Saúde Pública, v. 21, p. 525-34, 2005.

MINTZES, B. Disease mongering in drug promotion: do governments have a regulatory role? PLoS Medicine, v.3, n. 4, p. 461-5, 2006.

MINTZES, B. Head to head: Should patient groups accept money from drug companies? BMJ, v. 334, p. 935, 2007.

MOSCONI, P. Industry funding of patients' support groups. Declaration of competing interests is rare in Italian breast cancer associations. British Medical Journal, v.327, p.344, 2003.

PUBLIC CITIZEN. 2002 Drug Industry Profits: Hefty Pharmaceutical Company Margins Dwarf Other Industries. Congress Watch, June 2003. Disponível em: < http://www.citizen. org/documents/Pharma_Report.pdf>. Acesso em: 05 abr. 2011.

SANTOS, B.S. Saúde, justiça e democracia. In: SEMINÁRIO INTERNACIONAL DE DIREITO E SAÚDE, 10. jul. 2010, Rio de Janeiro. Informação verbal. Rio de Janeiro: ENSP, 2010. 
SILLUP, G.P.; PORTH, S.J. Ethical issues in the pharmaceutical industry: an analysis of US newspapers. International Journal of Pharmaceutical and Healthcare Marketing, v.2, n.3, p.163-180, 2008.

SOARES, J.C.R.S. O desafio da promoção do uso consciente e responsável de medicamentos frente ao forte apelo de consumo presente nas mensagens publicitárias e na mídia. Seminário Internacional de Propaganda de Medicamentos. ANVISA/OPAS, Brasília; abril 2005. Disponível em: <www.anvisa.gov.br/divulga/eventos/propaganda_medicamentos/ apresentacoes/jussara_reis_souza_soares.ppt> Acesso em: 05 abr. 2011.

SOARES, J.C.R.S. "Quando o anúncio é bom, todo mundo compra." O Projeto MonitorAÇÃO e a propaganda de medicamentos no Brasil. Ciência \& Saúde Coletiva, n.13(Sup), p.641-649, 2008.

SWEET, M. Pharmaceutical marketing and the internet. Australian Prescriber, v.32, p.2-4, 2009.

TEMPORÃO, J.G. O SUS e o direito da coletividade. Portal Saúde, Brasília, DF, 2009.

Disponível em: <http://portal.saude.gov.br/portal/arquivos/pdf/artigo_ministro.pdf>. Acesso em: 29 jul. 2009.

WEBB, S. Drug marketing and the new media. Nature Biotechnology, v.28, n.5, 2010.

WORLD HEALTH ORGANIZATION. World pharmaceutical sales and consumption. In: (Org). The world medicines situation. Geneva: WHO, 2004. Disponível em: < http://www.ops.org.bo/textocompleto/ime23901.pdf >. Acesso em: 11 jan. 2011.

Background document for the Intergovernmental Working Group on Public Health, Innovation and Intellectual Property (IGWG) process. Copenhagen: WHO, 2007. Disponível em: <http://www.patientsandpatents.com/us/resources/igwg_resources>. Acesso em: 16 mar 2011.

\section{Nota}

${ }^{1}$ JCRS Soares responsabilizou-se pela concepção do artigo, estruturação da discussão, revisão e aprovação da versão final do texto. AS Deprá e JCRS Soares realizaram os levantamentos bibliográficos, a discussão do conteúdo e a redação. Uma versão preliminar do artigo fundamentou a comunicação oral que foi apresentada no XXI Congresso da Associação Latina de Análise dos Sistemas de Saúde - CALASS 2010. 


\section{Abstract}

Dangerous liaisons: the pharmaceutical industry, patients associations and the legal battles for access to medicines

The main objective of this paper is to contribute to the debate on the legal claims for pharmaceutical drugs in Brazil, as part of the struggle for access to these products. A brief discussion about the pharma industry is made, followed by the state-of-the-art of the so-called process of "health judicialization". These are the basis for the reflections on the relationships between the corporations and patients' associations, which are part of the strategies used by the pharma industry in order to expand the market. These liaisons may be influencing the augmentation of court requests, thus damaging the responsible drug use. The role of commodification of healthcare is discussed.

Key words: pharmaceutical industry, patients' associations, drug utilization, judicial decisions. 